05,03

\title{
Общность процессов спонтанного и вынужденного перемагничивания кластеров MnSb, внедренных в тонкие пленки GaMnSb
}

\author{
(C) А.И. Дмитриев ${ }^{1}$, А.А. Филатов ${ }^{2}$ \\ ${ }^{1}$ Институт проблем химической фризики РАН, \\ Черноголовка, Россия \\ ${ }^{2}$ Московский государственный университет им. М.В. Ломоносова, \\ Москва, Россия \\ E-mail: aid@icp.ac.ru \\ (Поступила в Редакцию 24 января 2017 г. \\ В окончательной редакции 14 февраля 2017 г.)
}

Обнаружена общность процессов спонтанного и вынужденного перемагничивания кластеров $\mathrm{MnSb}$, внедренных в тонкие пленки GaMnSb. Родство термоактивационных и полевых процессов перемагничивания экспериментально выражается в том, что максимум на полевых зависимостях магнитной вязкости $S(H)$ совпадает с коэрцитивной силой $H_{C}$ образца. Анализ этого экспериментального факта позволил получить формулу, устанавливающую связь $H_{C}$ с параметрами модели, описывающей зависимости $S(H)$. Полученная формула идентична известному закону Кнеллера, определяющему температурную зависимость $H_{C}(T)$ невзаимодействующих суперпарамагнитных наночастиц.

Работа поддержана грантом Президента РФ МК-5754.2016.3.

DOI: 10.21883/FTT.2017.09.44841.012

\section{1. Введение}

В работах [1,2] было исследовано спонтанное перемагничивание (магнитная вязкость) тонких пленок $\mathrm{GaMnSb}$, содержащих кластеры MnSb. Интерес к этим образцам обусловлен спиновой поляризацией носителей заряда, индуцированной намагниченностью кластеров $\mathrm{MnSb}$ [3]. Зависимости магнитной вязкости от напряженности магнитного поля $S(H)$, соответствующей угловому коэффициенту временных зависимостей магнитного момента в полулогарифмических координатах, определяются логнормальным распределением энергии магнитной анизотропии кластеров $\mathrm{MnSb}$ [2]. Максимум кривых $S(H)$, построенных при различных температурах, совпадает с коэрцитивной силой образцов (рис. 1). В работе [2] этот экспериментальный факт не получил должного обсуждения и анализа. Настоящая работа направлена на то, чтобы восполнить этот пробел.

Ранее взаимосвязь спонтанного и вынужденного перемагничивания, выражающаяся в зависимости коэрцитивной силы $H_{C}$ от флуктуационного поля $H_{f}$, была установлена для постоянных магнитов семейства $\mathrm{NdFeB} \mathrm{[4].}$ В случае, когда оба рассматриваемых явления - магнитная вязкость и магнитный гистерезис - обусловлены смещением доменных стенок (механизм „слабого“ пиннинга), эта связь выражается простой линейной функцией $\lg H_{C}=\lg H_{f}+\lg \left(25\left(\left(31 \gamma b^{2} / 25 k_{\mathrm{B}} T\right)-1\right)\right)$ [4]. Здесь, $\gamma$ - поверхностная плотность энергии доменных стенок, $4 b$ - толщина доменной стенки, $k_{\mathrm{B}}-$ постоянная Больцмана, $T$ - температура. Флуктуационное поле есть псевдомагнитное поле, введенное Вольфартом, для обсуждения спонтанного перемагничивания, вызванного термическими флуктуациями. Физический смысл этой величины состоит в том, что $H_{f}$ является мерой энергии термических флуктуаций, выраженной в единицах напряженности магнитного поля. В настоящей статье рассматриваются процессы перемагничивания посредством вращения вектора намагничености кластеров $\mathrm{MnSb}-$ наночастиц с одноосной магнитной анизотропией, внедренных в тонкие пленки GaMnSb. В условиях иного механизма перемагничивания в отсутствие доменных стенок взаимосвязь спонтанного и вынужденного перемагничивания в кластерах $\mathrm{MnSb}$ может оказаться иной,

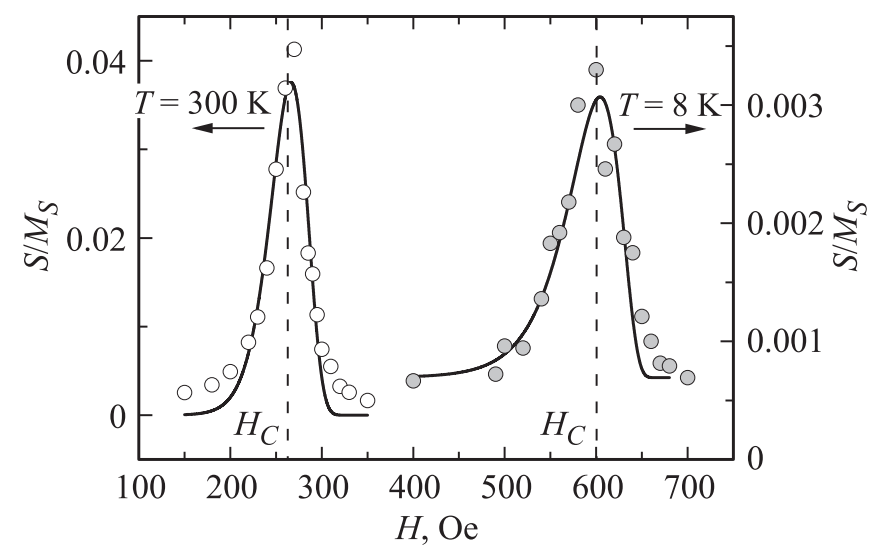

Рис. 1. Полевые зависимости магнитной вязкости образца при температурах $T=8 \mathrm{~K}$ и $300 \mathrm{~K}$. Вертикальными штриховыми линиями отмечены коэрцитивные силы при соответствующих температурах. 
чем в постоянных магнитах $\mathrm{NdFeB}$. Целями статьи являются: создание экспериментальных условий для поиска взаимосвязи термоактивационных и полевых процессов перемагничивания кластеров $\mathrm{MnSb}$, внедренных в тонкие пленки GaMnSb, а также установление механизмов этой взаимосвязи.

\section{2. Методика}

Пленки $\mathrm{GaMnSb}$ толщиной $\sim 90 \mathrm{~nm}$ были получены из системы $\mathrm{GaSb}-\mathrm{MnSb}$ эвтектического состава, методом импульсного лазерного осаждения. В качестве подложек использовался монокристаллический $\mathrm{Al}_{2} \mathrm{O}_{3}$. Структура и состав тонких пленок контролировались сканирующим зондовым микроскопом и сканирующим электронным микроскопом. С помощью магнитно-силового микроскопа было установлено, что пленки содержат кластеры $\mathrm{MnSb}$ средним диаметром $D=55 \mathrm{~nm}$ [2]. Химический состав приповерхностных слоев пленок был определен методом энергодисперсионной спектроскопии. Подробно методика изготовления и аттестации образцов описана ранее [5].

С помощью СКВИД-магнитометра (MPMS 5XL Quantum Design) были выполнены измерения зависимостей магнитного момента образцов $M$ от температуры $T$ и напряженности магнитного поля $H$ (в виде петель гистерезиса при различных температурах).

\section{3. Результаты и обсуждение}

3.1. Зависимость магнитной вязкости от напряженности магнитного поля. Магнитная вязкость тонких пленок $\mathrm{GaMnSb}$, содержащих кластеры $\mathrm{MnSb}$, подробно была исследована нами ранее в работах $[1,2]$. Здесь кратко приведены лишь те экспериментальные результаты, которые необходимы для обсуждения взаимосвязи спонтанного и вынужденного перемагничивания образцов. На рис. 1 представлены полевые зависимости магнитной вязкости $S(H)$ образца при температурах $T=8 \mathrm{~K}$ и $T=300 \mathrm{~K}$. Вертикальными пунктирными линиями отмечены коэрцитивные силы при соответствующих температурах (будут подробно обсуждаться ниже). На рис. 1 сплошными линиями показаны аппроксимации зависимостей $S(H)$ функцией [2]

$$
\frac{S}{M_{S}}=\frac{t}{25(1-h)^{2}} \frac{1}{\sqrt{2 \pi \sigma}} \exp \left(-\frac{1}{2}\left(\frac{\ln \frac{t}{(1-h)^{2}}}{\sigma}\right)^{2}\right)
$$

где $h=H / H_{A}, t=T / T_{B}$ (здесь $T_{B}$ - температура блокировки кластеров), $M_{S}$ - намагниченность насыщения, $\sigma$ - дисперсия магнитной анизотропии. Из аппроксимации зависимости $S(H)$ при температуре $T=8 \mathrm{~K}$ были определены температура блокировки кластеров $T_{B}=390 \mathrm{~K}$, поле магнитной анизотропии $H_{A}=700$ Ое и ее дисперсия $\sigma=1.9$.

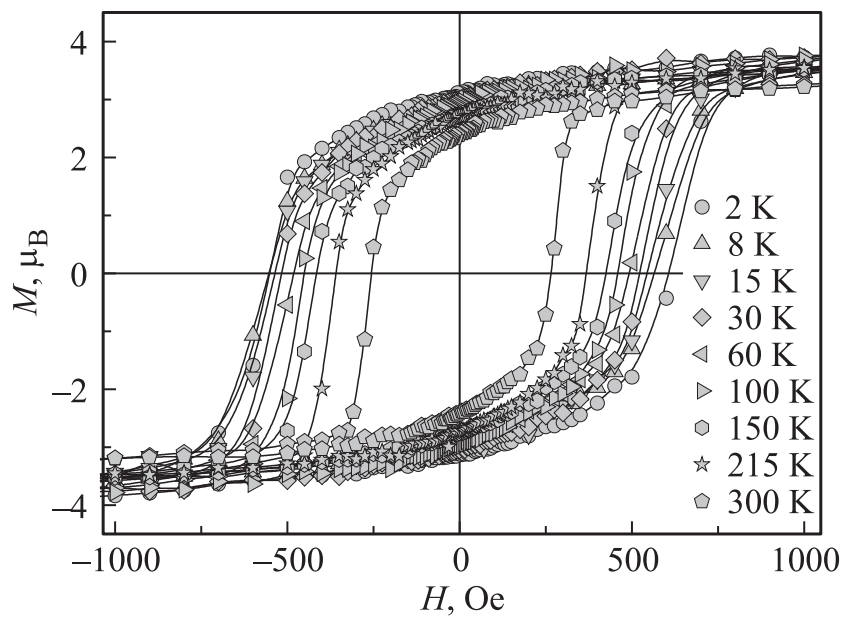

Рис. 2. Петли магнитного гистерезиса образца при различных температурах.

3.2. Взаимосвяз спонтанного и вынужденного перемагничивания. Температурная зависимость коэрцитивной силы. Из рис. 1 следует, что максимум кривых $S(H)$ совпадает с коэрцитивной силой $H_{C}$ образца. Значение напряженности магнитного поля $H$, при котором функция (1) достигает максимума, как следует из рис. 1 , есть коэрцитивная сила $H_{C}$. Таким образом, для установления связи коэрцитивной силы $H_{C}$ с параметрами модели (1) необходимо найти экстремум функции (1)

$$
\begin{aligned}
\frac{\partial S}{\partial H}= & \frac{\frac{t}{25 \sigma} \sqrt{\frac{2}{\pi}} \exp \left(-\frac{\ln ^{2}\left(\frac{t}{(1-h)^{2}}\right)}{2 \ln ^{2} D}\right)}{H_{A}(1-h)^{3}} \\
& -\frac{\frac{t}{25 \sigma} \sqrt{\frac{2}{\pi}} \ln \left(\frac{t}{(1-h)^{2}}\right) \exp \left(-\frac{\ln ^{2}\left(\frac{t}{(1-h)^{2}}\right)}{2 \ln ^{2} D}\right)}{H_{A} \ln ^{2} D(1-h)^{3}}=0 .
\end{aligned}
$$

Решением уравнения (2) является выражение

$$
H_{C}=H_{A}\left(1-\sqrt{\frac{T}{T_{B} \sigma^{\ln \sigma}}}\right) .
$$

Выражение (3) устанавливает связь коэрцитивной силы $H_{C}$ с параметрами модели (1), которая в явном виде не содержит $H_{C}$. Другими словами, уравнение (3) и есть выражение искомой взаимосвязи полевых процессов вынужденного перемагничивания кластеров $\mathrm{MnSb}$, внедренных в тонкие пленки GaMnSb, и термоактивационных процессов их спонтанного перемагничивания.

Для проверки справедливости формулы (3), описывающей температурную зависимость коэрцитивной силы $H_{C}$, были измерены петли магнитного гистерезиса при различных температурах (рис. 2). Полученные гистерезисные кривые типичны для ферромагнетиков: 


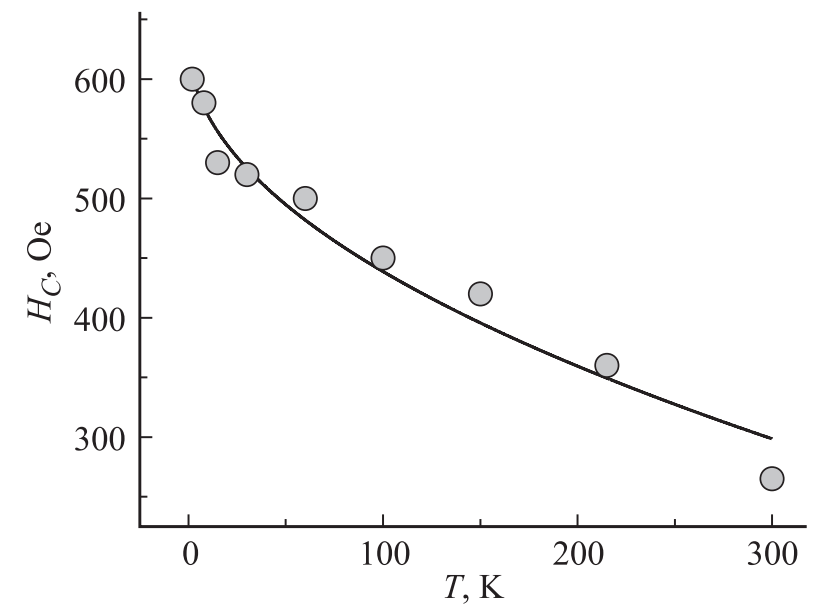

Рис. 3. Температурная зависимость коэрцитивной силы образца. Сплошной линией показана аппроксимация.

наблюдается насыщение в магнитных полях $\sim 500 \mathrm{Oе}$, форма петель близка к прямоугольной, коэрцитивная сила сильно зависит от температуры. Зависимость $H_{C}(T)$ показана на рис. 3. С понижением температуры коэрцитивная сила увеличивается от 260 Ое при комнатной температуре до 600 Ое при температуре $T=2$ K. Сплошной линией на рис. 3 показана аппроксимация зависимости $H_{C}(T)$ выражением (3). На рис. 3 видно, что кривая, задаваемая формулой (3), достаточно близко воспроизводит экспериментальные точки. Из аппроксимации зависимости $H_{C}(T)$ выражением (3) были определены температура блокировки кластеров $T_{B}=430 \pm 40 \mathrm{~K}$, поле магнитной анизотропии $H_{A}=630 \pm 70 \mathrm{Oе}$ и ее дисперсия $\sigma=1.5 \pm 0.4$, которые оказались близки к соответствующим значениям, определенным из аппроксимации зависимостей $S(H)$ выражением (1).

Следует отметить, что формула, определяющая зависимость $H_{C}(T)$ для невзаимодействующих суперпарамагнитных наночастиц, подобная (3), впервые была получена в работе [6]. Она носит имя Кнеллера. Однако выражение (3), в отличие от формулы Кнеллера, учитывает распределение наночастиц по размерам. Ранее было установлено, что закон Кнеллера применим к самым различным наночастицам: $\mathrm{Fe}$ [7], $\mathrm{Cu}_{x} \mathrm{Co}_{1-x}$ [8], $\mathrm{ZnFe}_{2} \mathrm{O}_{4}$ [9], $\mathrm{CoFe}_{2} \mathrm{O}_{4}$ [10,11], $\mathrm{NiFe}_{2} \mathrm{O}_{4}$ [12], $\mathrm{FeC}$ [13], $\mathrm{Mn}_{0.5} \mathrm{Zn}_{0.5} \mathrm{Gd}_{0.02} \mathrm{Fe}_{1.98} \mathrm{O}_{4}[14], \mathrm{Sr}_{1 / 3} \mathrm{Mn}_{1 / 3} \mathrm{Co}_{1 / 3} \mathrm{Fe}_{2} \mathrm{O}_{4}[15]$ и др. При этом в работах $[9,12,14]$ были обнаружены расхождения между экспериментальными зависимостями $H_{C}(T)$ и формулой Кнеллера, которые объясняются несколькими факторами: в первую очередь распределением наночастиц по размерам. Также диполь-дипольным взаимодействием между наночастицами, неупорядоченностью поверхностных спинов, которые влияют на поверхностную анизотропию и, следовательно, суммарную анизотропию наночастиц, разбросом осей легкого намагничивания и т.д. Как видно из рис. 3, формула (3), учитывая распределение кластеров по размерам, удовлетворительно воспроизводит экспериментальные точки. Более того, значения дисперсии $\sigma$ и температуры блокировки $T_{B}$ (соответствующей медиане функции распределения кластеров по размерам $f(D)$ ), определенные из аппроксимации зависимостей $H_{C}(T)$ формулой (3), позволяют восстановить вид функции $f(D)$.

3.3. Температурная зависимость магнитного момента. На рис. 4 приведена температурная зависимость магнитного момента образца $M(T)$. При понижении температуры наблюдается рост величины $M$. В интервале температур $T=30-300 \mathrm{~K}$ кривая выпукла. При температуре $T=30 \mathrm{~K}$ происходит перегиб кривой, так что в интервале температур $T=2-30 \mathrm{~K}$ кривая вогнута. Высокотемпературный участок кривой (при $T>30 \mathrm{~K}$ ) описывался функцией $M(T)=M(0)\left[1-\left(T / T_{C}\right)^{\alpha}\right]$, где $M(0)$ - намагниченность при $T=0 \mathrm{~K}, T_{C}-$ температура Кюри. При $\alpha=3 / 2$ указанная зависимость называется законом Блоха („законом 3/2“), которым хорошо описываются низкотемпературные $\left(T<0.5 T_{C}\right)$ фрагменты зависимостей $M(T)$ массивных ферромагнитных образцов. Закон Блоха является следствием возбуждения длинноволновых спиновых волн - магнонов, число которых при низких температурах растет пропорционально $T^{3 / 2}$. Из аппроксимации высокотемпературной части зависимости $M(T)$, показанной на рис. 4 сплошной линией, была определена температура Кюри $T_{C}=650 \pm 60 \mathrm{~K}$, которая оказалась близка к известному значению $590 \mathrm{~K}$ для соединения $\mathrm{MnSb}[16]$, и значение показателя степени $\alpha=2.4 \pm 0.2$. Известно, что для наночастиц изза эффектов ограничения размера наблюдаются отклонения от „закона $3 / 2$ “. Так, например, для наночастиц $\mathrm{CoFe}_{2} \mathrm{O}_{4}$ [11], $\mathrm{NiFe}_{2} \mathrm{O}_{4}$ [12], $\mathrm{FeC}$ [13], $\mathrm{CuFe}_{2} \mathrm{O}_{4}$ [17], $\gamma-\mathrm{Fe}_{2} \mathrm{O}_{3}$ и $\mathrm{Fe}_{3} \mathrm{O}_{4}[18]$ имело место значение $\alpha=2$. Ограничение размера наночастиц приводит к тому,

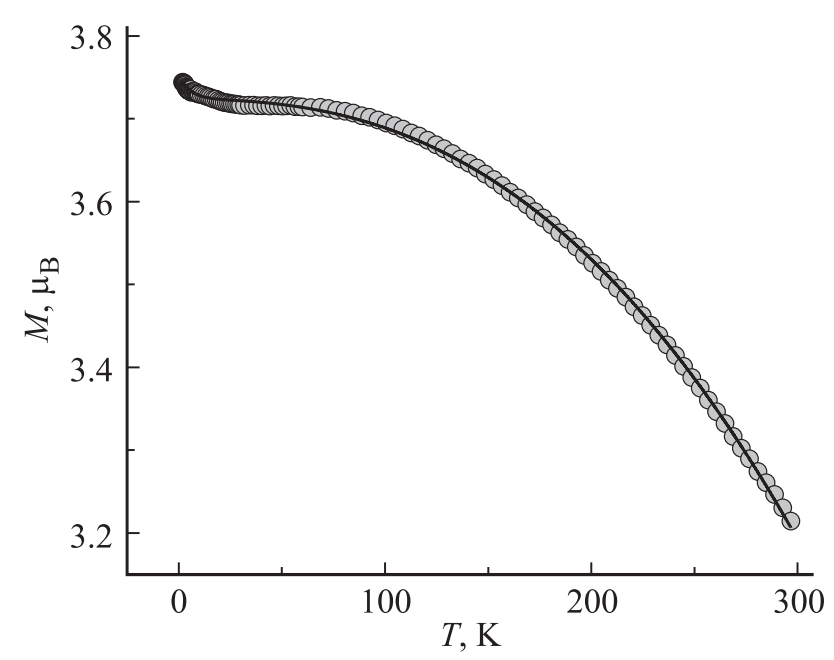

Рис. 4. Температурная зависимость магнитного момента образца. Сплошной линией показана аппроксимация. 
что магноны с длиной волны, превосходящей размеры наночастицы, не могут возбуждаться, что формирует энергетическую щель в дискретном спектре спиновых волн $[14,19,20]$. Другая причина заключается в обрыве обменных связей у поверхностных атомов, что приводит к фрустрированным магнитным состояниям и спиновому беспорядку.

Ранее перегиб на зависимостях $M(T)$, аналогичный тому, который наблюдается при $T=30 \mathrm{~K}$ в наших экспериментах, наблюдался на температурных зависимостях магнитного момента наночастиц $\mathrm{Sr}_{1 / 3} \mathrm{Mn}_{1 / 3} \mathrm{Co}_{1 / 3} \mathrm{Fe}_{2} \mathrm{O}_{4}$ [15], $\mathrm{CoFe}_{2} \mathrm{O}_{4}$ [21], $\mathrm{CuFe}_{2} \mathrm{O}_{4}$ и $\mathrm{MnFe}_{2} \mathrm{O}_{4}$ [22]. Эту особенность, как правило, относят к неупорядоченным поверхностным спинам. Их вклад в температурную зависимость суммарного магнитного момента описывался феноменологическим выражением $A \exp \left(-T / T_{f}\right)$, где $A$ - доля приповерхностных атомов, $T_{f}$ - температура замерзания термически индуцированных флуктуаций поверхностных спинов [22]. Из аппроксимации низкотемпературной части зависимости $M(T)$, показанной на рис. 4 сплошной линией, была определена температура замерзания $T_{f}=8 \mathrm{~K}$. Соответствующая энергия $k_{\mathrm{B}} T_{f} \sim 1 \cdot 10^{-15} \mathrm{erg}$ оказалась близка к значению обменного интеграла $7 \cdot 10^{-15} \mathrm{erg}$ в тонких пленках $\mathrm{MnSb}$ [23].

\section{4. Выводы}

Измерены зависимости магнитного момента тонких пленок $\mathrm{GaMnSb}$, содержащих кластеры $\mathrm{MnSb}$, от температуры и напряженности магнитного поля (в виде петель гистерезиса при различной температуре). Температурная зависимость коэрцитивной силы $H_{C}(T)$ кластеров $\mathrm{MnSb}$ описывается модифицированным законом Кнеллера, учитывающим распределение кластеров по размерам $f(D)$. Анализ экспериментальной кривой $H_{C}(T)$ позволил определить температуру блокировки кластеров $T_{B}=430 \mathrm{~K}$, поле магнитной анизотропии $H_{A}=630$ Ое и дисперсию функции $f(D) \sigma=1.5$.

Температурная зависимость магнитного момента $M(T)$ образцов описывается модифицированным законом Блоха. Отклонение от ,закона 3/2“ обусловлено ограничением размера кластеров $\mathrm{MnSb}$, которое приводит к подавлению возбуждения магнонов с длиной волны, превосходящей размеры кластеров. При температуре $T=30 \mathrm{~K}$ на зависимости $M(T)$ обнаружен перегиб, обусловленный вкладом в суммарный магнитный момент неупорядоченных поверхностных спинов. Этот вклад становится заметным при температурах, сопоставимых с температурой замерзания $T_{f}=8 \mathrm{~K}$ термически индуцированных флуктуаций поверхностных спинов.

А.И. Дмитриев признателен за предоставленные образцы Р.Б. Моргунову - учителю, многолетней работой с которым обусловлен интерес к теме, обсуждаемой в статье, и А.Д. Таланцеву за помощь в исследованиях на СКВИД-магнитометре.

\section{Список литературы}

[1] А.И. Дмитриев, А.А. Филатов. ФТТ 58, 1935 (2016).

[2] A.I. Dmitriev, A.D. Talantsev, O.V. Koplak, R.B. Morgunov. J. Appl. Phys. 119, 073905 (2016).

[3] В.В. Рыльков, Б.А. Аронзон, Ю.А. Данилов, Ю.Н. Дроздов, В.П. Лесников, К.И. Маслаков, В.В. Подольский. ЖЭТФ 127, 838 (2005).

[4] J. Liu, S. Pan, H. Luo, D. Hou, X. Nie. J. Phys. D 24, 384 (1991).

[5] С.Ф. Маренкин, О.А. Новодворский, А.В. Шорохова, А.Б. Давыдов, Б.А. Аронзон, А.В. Кочура, И.В. Федорченко, О.Д. Храмова, А.В. Тимофеев. Неорган. материалы 50, 973 (2014).

[6] E.F. Kneller, F.E. Luborsky. J. Appl. Phys. 34, 656 (1963).

[7] A.K. Giri. J. Appl. Phys. 81, 1348 (1997).

[8] W.C. Nunes, W.S.D. Folly, J.P. Sinnecker, M.A. Novak. Phys. Rev. B 70, 014419 (2004).

[9] E.C. Mendonca, C.B.R. Jesus, W.S.D. Folly, C.T. Meneses, J.G.S. Duque, A.A. Coelho. J. Appl. Phys. 111, 053917 (2012).

[10] M.H. Carvalho, R.J.S. Lima, C.T. Meneses, W.S.D. Folly, V.H.V. Sarmento, A.A. Coelho, J.G.S. Duque. J. Appl. Phys. 119, 093909 (2016).

[11] K. Maaz, M. Usman, S. Karim, A. Mumtaz, S.K. Hasanain, M.F. Bertino. J. Appl. Phys. 105, 113917 (2009).

[12] K. Maaz, A. Mumtaz, S.K. Hasanain, M.F. Bertino. J. Magn. Magn. Mater. 322, 2199 (2010).

[13] S. Linderoth, L. Balcells, A. Labarta, J. Tejada, P.V. Hendriksen, S.A. Sethi. J. Magn. Magn. Mater. 124, 269 (1993).

[14] I.M. Obaidat, B. Issa, B.A. Albiss, Y. Haik. IOP Conf. Ser. Mater. Sci. Eng. 92, 012012 (2015).

[15] N.S.E. Osman, T. Moyo. J. Supercond. Nov. Magn. 29, 361 (2016).

[16] R. Coehoorn, C. Haas, R.A. Groot. Phys. Rev. B 31, 1980 (1985).

[17] C.R. Alves, R. Aquino, M.H. Sousa, H.R. Rechenbrg, G.F. Goya, F.A. Tourinho, J. Depeyrot. J. Met. Nanocryst. Mater. 20-21, 694 (2004).

[18] D. Ortega, E. Vélez-Fort, D.A. García, R. García, R. Litrán, C. Barrera-Solano, M. Ramírez-del-Solar, M. Domínguez. Phil. Trans. R. Soc. A 368, 4407 (2010).

[19] P.V. Hendriksen, S. Linderoth, P.A. Lindgard. J. Magn. Magn. Mater. 104-107, 1577 (1992).

[20] P.V. Hendriksen, S. Linderoth, P.A. Lindgard. Phys. Rev. B 48, 7259 (1993).

[21] C.R. Alves, R. Aquino, J. Depeyrot, T.A. Cotta, M.H. Sousa, F.A. Tourinho, H.R. Rechenberg, G.F. Goya. J. Appl. Phys. 99, 08M905 (2006).

[22] R. Aquino, J. Depeyrot, M.H Sousa, F.A. Tourinho, E. Dubois, R. Perzynski. Phys. Rev. B 72, 18443 (2005).

[23] M. Hashimoto. J. Phys. Soc. Jpn. 22, 869 (1967). 